Cahiers $d u$ MONDE RUSSE

\section{Cahiers du monde russe}

Russie - Empire russe - Union soviétique et États indépendants

$43 / 2-3 \mid 2002$

Contacts intellectuels, réseaux, relations internationales

\title{
Le contexte européen (français et allemand) du formalisme russe
}

\section{Aleksander DMITRIEV}

\section{(2) OpenEdition}

Journals

Édition électronique

URL : https://journals.openedition.org/monderusse/8505

DOI : 10.4000/monderusse. 8505

ISSN : $1777-5388$

Éditeur

Éditions de l'EHESS

\section{Édition imprimée}

Date de publication : 1 avril 2002

Pagination : 423-440

ISBN : 2-7132-1781-4

ISSN : $1252-6576$

Référence électronique

Aleksander DMITRIEV, « Le contexte européen (français et allemand) du formalisme russe ", Cahiers du monde russe [En ligne], 43/2-3 | 2002, mis en ligne le 01 janvier 2007, consulté le 04 septembre 2022. URL : http://journals.openedition.org/monderusse/8505 ; DOI : https://doi.org/10.4000/ monderusse.8505 


\title{
CAIR N
}

chercher : repérer : avancer

Cet article est disponible en ligne à l'adresse :

http://www.cairn.info/article.php?ID REVUE=CMR\&ID NUMPUBLIE=CMR 432\&ID ARTICLE=CMR 4320423

\section{Le contexte européen (Français et Allemand) du formalisme russe}

\author{
par ALEKSANDR DMITRIEV
}

| Editions de l'EHESS | Cahiers du monde russe

2002/2-3 - Vol 43

ISSN 1252-6576 | ISBN 2713217814 | pages 423 à 440

Pour citer cet article :

- DMITRIEV A., LE CONTEXTE EUROPÉEN (FRANÇAIS ET ALLEMAND) DU FORMALISME RUSSE, Cahiers du monde russe 2002/2-3, Vol 43, p. 423-440.

Distribution électronique Cairn pour les Editions de l'EHESS.

(C) Editions de l'EHESS. Tous droits réservés pour tous pays.

La reproduction ou représentation de cet article, notamment par photocopie, n'est autorisée que dans les limites des conditions générales d'utilisation du site ou, le cas échéant, des conditions générales de la licence souscrite par votre établissement. Toute autre reproduction ou représentation, en tout ou partie, sous quelque forme et de quelque manière que ce soit, est interdite sauf accord préalable et écrit de l'éditeur, en dehors des cas prévus par la législation en vigueur en France. Il est précisé que son stockage dans une base de données est également interdit. 


\section{LE CONTEXTE EUROPÉEN (FRANÇAIS ET ALLEMAND) DU FORMALISME RUSSE*}

L'école formaliste représente une des avancées les plus importantes et les plus originales qui se soient produites dans le domaine des sciences humaines russes ${ }^{1}$. En même temps, son histoire ne peut être dissociée de la science européenne, au premier chef allemande et française, de son époque et doit être située dans ce contexte qui permettra de mieux évaluer les innovations et les particularités de cette école. L'influence de la philologie occidentale, l'attention qu'on lui prêtait en Russie, furent loin d'être uniformes au sein de l'« école formelle » comme dans les courants qui lui étaient proches et elles ne demeurèrent pas statiques au cours des années 1910-1920. Enfin le formalisme ne fut largement reconnu (par la parution de traductions, d'anthologies, de recueils, de travaux qui lui furent consacrés) qu'au cours des années 1960, soit plusieurs décennies après son extinction à la charnière des années 20 et 30 . Un tel décalage chronologique n'est pas chose fréquente dans la vie scientifique, surtout au XXe siècle, et c'est pourquoi il mérite d'être analysé. Ces questions ne peuvent être abordées dans le cadre exclusif de l'histoire d'une discipline, car les connexions internationales du formalisme ont un rapport direct avec l'histoire politique de la science, plus particulièrement avec celle des relations scientifiques entre l'URSS et l'étranger au cours du premier tiers du XXe siècle.

Sur le plan méthodologique, le postulat de base des formalistes - l'analyse de la chose littéraire « en tant que telle », indépendamment de ses sources biographiques, sociales ou idéologiques - s'appuyait sur une tradition européenne, dans

* Cette recherche a bénéficié de l'aide financière de la Gerda-Henkel-Stiftung (Programm zur Förderung des Historikernachwuchses in Russland), projet 01/SR/02.

Nous voudrions remercier E. V. Dušečkina et D. V. Ustinova qui nous ont permis de travailler sur certaines sources utilisées dans cet article.

1. Comme c'est avec l'école formaliste que commença précisément une nouvelle étape dans l'interpénétration des études littéraires et de la linguistique, nous aborderons également cette dernière dans notre étude. Nous continuerons d'appeler «philologie » (filologija) le domaine qui réunissait traditionnellement en Russie ces deux branches de recherche, mais nous attacherons une attention particulière à l'étude de la littérature. 
laquelle la branche russe des études littéraires s'inscrivait de façon assez organique. Lorsqu'Aleksandr N. Veselovskij formulait les bases de sa poétique historique, il comparait déjà l'étude de la littérature à un territoire «sur lequel l'historien littéraire chasse aux côtés de l'historien de l'art, du philologue et du sociologue » et insistait sur l'identité méthodologique particulière du premier ${ }^{2}$. Dans cette quête, Veselovskij s'appuyait en grande partie sur l'héritage scientifique de l'historien littéraire allemand Wilhelm Scherer, proche du positivisme ${ }^{3}$. En même temps, les héritiers de Veselovskij (Aleksandr N. Pypin, Fedor I. Buslaev et d'autres), qui furent des adeptes de la méthode d'histoire culturelle, s'en tenaient à l'idée que la littérature se dissolvait dans la vie culturelle de la société. Parmi les prédécesseurs importants du formalisme, on peut également citer Ferdinand Brunetière, dont l'idée d'une évolution autonome des genres littéraires, et par conséquent de leur analyse spécifique, fut reprise par Viktor Šklovskij dans son article programmatique «Svjaz' priemov sjužetosloženija s obščim priemami stilja » (Le lien entre les procédés de construction du sujet et les procédés stylistiques généraux) (1919)4. Gustave Lanson, adversaire politique et idéologique de Brunetière, ce qui ne l'avait pas empêché de beaucoup puiser chez lui, défendait le principe d'une approche historique dans l'étude de la littérature, tout en soulignant les logiques indépendantes qui présidaient à l'évolution littéraire et qu'il fallait préserver selon lui contre tout réductionnisme psychologique, sociologique ou autre ${ }^{5}$. Mihail Geršenzon qui, dans ses propres travaux, s'appuyait plutôt sur la tradition de la Geistesgeschichte et qui cultivait le genre de l'essai philosophique, écrivit en 1911 une postface très conceptuelle au livre de Lanson, dans laquelle, tout à fait dans la logique de Veselovskij, il prônait une indépendance des études littéraires ${ }^{6}$. C'est pourtant la tradition philologique allemande qui offrit à l'école formelle ses bases et ses formulations décisives au milieu des années 1910. Voyons cela de plus près :

- Dans le domaine philosophique, c'est incontestablement la tradition kantienne qui fut particulièrement importante, aussi bien dans sa variante orthodoxe et « scolaire », par l'intermédiaire d'A. I. Vvedenskij, professeur à l'université de

2. Cité d'après B. Erlih, Russkij formalizm : istorija i teorija (Le formalisme russe : histoire et théorie), Saint-Pétersbourg, Akademičeskij proekt, 1996, p. 27.

3. Voir A. V. Mihajlov, Problemy istoričeskoj poetiki v istorii nemeckoj kul'tury. Očerki iz istorii filologičeskoj nauki (Problèmes de la poétique historique dans l'histoire de la culture allemande. Essais d'histoire de la science philologique), Moscou, Nauka, 1989, p. 205-212.

4. S. L. Kozlov, « Literaturnaja evoljucija i literaturnaja revoljucija : k istorii idej » (Evolution et révolution littéraires: contribution à une histoire des idées », in Tynjanovskij sbornik. Četvertye tynjanovskie čtenija (Recueil Tynjanov, Quatrième recontre Tynjanov), Riga, Zinathe, 1990,p. 116-118. Dans Russkaja mys1', n 7 et 8, 1891, on trouve un compte rendu détaillé du livre de Brunetière, $L$ 'évolution des genres dans l'histoire de la littérature française, paru en 1891.

5. Sur cette question des liens entre Lanson et Brunetière, voir Antoine Compagnon, La troisième république des lettres, de Flaubert à Proust, Paris, Seuil, 1983, p. 175-177.

6. M. Geršenzon, postface à G. Lanson, Metod v istorii literatury (La méthode en histoire de la littérature), Moscou, Mir, 1911. Da façon significative, V. M. Žirmunskij cite ce texte dans son écrit programmatique Zadači poetiki (Les tâches de la poétique). 
Saint-Pétersbourg ${ }^{7}$, que dans les recherches d'une indépendance méthodologique des « sciences de l'esprit » (à cet égard l'influence de Heinrich Rickert fut essentielle ${ }^{8}$. Plus tard, Roman Jakobson allait tracer un lien entre les tendances antipsychologiques de la phénoménologie de Husserl et l'école formelle naissante, dès avant la naissance de la Société d'étude du langage poétique (OPOJaZ - Obščestvo po izučeniju poetičeskogo jazyka) en 19179 . J. Toman, qui a consacré une recherche précise à cette question, conclut toutefois qu'il ne s'agissait pas ici d'un emprunt de tel ou tel procédé phénoménologique par la nouvelle philologie (ce qui arriva en partie plus tard dans l'itinéraire de Jakobson au sein du Cercle linguistique de Prague), mais d'une approche conceptuelle commune, visant à fonder un regard «immanent» sur l'activité spirituelle de l'homme ${ }^{10}$.

- Parmi les domaines voisins des études littéraires, l'expérience la plus importante pour la genèse du formalisme fut celle de l'histoire de l'art allemande, avant tout de l'école de Heinrich Wölfflin, avec son idée d'une histoire « anonyme » de l'art (dans la revue Letopis', Viktor Šklovskij publia en 1916 un compte rendu positif de l'ouvrage, traduit en russe, de Karl Voll, Vergleichende Gemäldestudien) ${ }^{11}$. En

7. M. O. Čudakova et E. A. Toddes, « Tynjanov v vospominanijah sovremennikov» (Tynjanov dans les mémoires de ses contemporains), Pervye tynjanovskie čtenija (Première rencontre Tynjanov), Riga, Zinatne, 1986, p. 93 (il s’agit des mémoires de Jurij Oksman) ; Catherine Depretto, « Peterburgskij universitet i Serebrjanyj vek » (L'université de Saint-Pétersbourg et l'Âge d'argent), in Sankt-Peterburg : okno v Rossiju. Gorod, modernizacija, sovremennost' (Saint-Pétersbourg, une fenêtre vers la Russie. Ville, modernisation, modernité), Saint-Pétersbourg, Feniks, 1997, p. 87.

8. En janvier 1919, Boris M. Ejhenbaum fit un cours de méthodologie sur les Sciences de la nature et sciences de la culture de Rickert, comme en témoigne son journal du 12 au 18 janvier 1919 ; cité d'après Ju. L. Tynjanov, Poetika, istorija literatury, kino (Poétique, histoire littéraire, cinéma), Moscou, Nauka, 1977, p. 455.

9. Voir R. Jakobson, K. Pomorska, Besedy (Entretiens), Jérusalem, Ed. I. L. Magnes, 1982, p. 10, et la monographie d'E. Holenstein, Roman Jakobsons phänomenologischer Strukturalismus, Francfort-sur-le-Main, 1975.

10. J. Toman, The magic of a common language, Jakobson, Mathesius, Trubetskoy and the Prague Linguistic Circle, Cambridge (Mass.) - Londres, MIT Press, 1995, p. 30-34. On retrouve ce désir de fonder philosophiquement l'autonomie de l'objet esthétique (y compris, en l'occurrence, littéraire), à la manière de l'esthétique de Richard Hamann, chez B. M. Engel'gardt dans les années 1910 ; il avait fréquenté le séminaire de S. A. Vengerov avec Tynjanov, puis devint collègue de ce dernier à l'Institut d'histoire des arts et auteur d'un ouvrage profond, Formal'nyj metod $v$ istorii literatury (La méthode formelle dans l'histoire littéraire, 1927). Voir A. B. Muratov, Fenomenologičeskaja estetika načala $X X$ veka i teorija slovesnosti (B.M. Engel'gardt) (L'esthétique phénoménologique du début du $X X^{\mathrm{e}}$ siècle, B. M. Engel'gardt), Saint-Pétersbourg, Ed. de l'Université, 1996.

11. Voir aussi les revues bibliographiques de B. L. Bogaevskij, «Zadači iskusstvoznanija » (Les tâches de l'histoire de l'art), in Zadači i metody izučenija iskusstv (Les tâches et les méthodes dans l'étude des arts), Petrograd, 1924 (volume programmatique de l'Institut d'histoire des arts ; A. Sidorov, « Očerk literatury po iskusstvovedeniju 1915-1919 » (Revue bibliographique en histoire de l'art 1915-1919), Naučnye izvestija Akademičeskogo centra Narkomprosa, 2, 1922. Il faut surtout mentionner la réception en Russie du livre important de Max Dessoir, Zeitschrift für Ästhetik und allgemeine Kunstwissenschaft, et la traduction du livre d'Adolf von Hilderbrand, Das Problem der Form in der bildenden Kunst (Problema formy v izobrazitel'nom iskusstve, Moscou, 1914). 
janvier 1919, Ejhenbaum notait dans son journal à propos des Kunstgeschichtliche Grundbegriffe de Wölfflin, qu'il lisait alors : «On pense constamment à des analogies avec la construction d'une histoire de la littérature. Il faut mettre au point un système de concepts fondamentaux et soumettre la littérature à une analyse, dans laquelle tel ou tel phénomène soit examiné $d$ 'un seul point de vue, en épuisant tous les moyens dont on dispose. $»^{12}$

- Dans le domaine de l'histoire littéraire, il faut citer une étude proche des recherches formalistes et qui fut l'analyse par Wilhelm Dibelius de la technique narrative de Dickens (il s'agit plutôt, en l'occurrence, d'un intérêt commun pour les procédés littéraires, et non pas d'une unité de méthode) ${ }^{13}$. Lorsque, dans son ouvrage consacré à Gogol et à l'école «naturaliste », V. V. Vinogradov analyse l'article d'Ejhenbaum «Kak sdelana 'Šinel'" Gogolja (Comment est fait Le Manteau de Gogol), il note l'influence sur Ejhenbaum de l'œuvre de Käte Friedemann sur le rôle du narrateur dans l'art de la prose ${ }^{14}$. Dans leur volonté de dépasser la théorie de Potebnja sur les images dans l'art littéraire, les formalistes et les philologues qui en étaient proches (Tynjanov, Žirmunskij) utilisèrent les travaux de Theodor Meyer sur la perceptibilité spécifique, non visuelle, de la poésie ${ }^{15}$.

- Dans leurs recherches sur le langage poétique, les formalistes tinrent le plus grand compte des acquis de la «philologie acoustique » (Ohrenphilologie) des Allemands Eduard Sievers et Franz Saran ${ }^{16}$. À la différence de la traditionnelle « philologie du texte », ceux-ci avaient cherché à découvrir par la voie expérimentale (en utilisant les moyens techniques dont ils pouvaient disposer à l'époque) les normes sonores des vers ainsi que les particularités de leur lecture par les auteurs ${ }^{17}$. Dès le premier volume du Sbornik po teorii poetičeskogo jazyka (Recueil de théorie du langage poétique ) paru en 1916, Vladimir Šklovskij, le frère aîné de Viktor et élève de V.F. Šišmarev, publia des comptes rendus de ces travaux

12. Cité d'après B. Ejhenbaum, O literature (De la littérature), Moscou, Sovetskij pisatel', 1988, p. 511.

13. Dans son Molodoj Tolstoj (Le jeune Tolstoj, 1921), Ejhenbaum, en même temps que Šklovskij, se réfère à Dibelius et à son analyse de Sterne dans Englische Romankunst (1911), ibid., p. 133-134. Dans leur étude de la composition des nouvelles, des auteurs moscovites tels que M. A. Petrovskij et A. A. Reformackij s'inspiraient également en grande partie de Dibelius.

14. V. V. Vinogradov, Gogol' i natural'naja škola (Gogol et l'école naturaliste), Leningrad, 1925, p. 13 (K. Friedemann, Die Rolle des Erzählers, Leipzig, 1910).

15. V. M.Žirmunskij, Teorija literatury, Poetika, Stilistika (Théorie de la littérature, Poétique, Stylistique), Moscou, Nauka, 1977, p. 20 ; Th. Meyer, Das Stilgesetz der Poesie, Leipzig, 1901.

16. En 1912-1913, F. A. Braun conseillait dans ses lettres à Žirmunskij, qui séjournait alors en Allemagne et qui se passionnait pour l'interprétation philosophique symboliste du romantisme de fréquenter les conférences de Sievers et de Saran ; voir PFA RAN (Archives de l'Académie des sciences de Russie, Section de Saint-Pétersbourg), f. 1001, op. 3, d. 232,1. 10 ob. Pour une présentation bibliographique générale de cette question, faite par un des « jeunes formalistes », voir N. A. Kovarskij, « Melodika stiha » (La mélodique des vers), Poetika, 4, Leningrad, 1928.

17. Cette direction de recherche fut développée au cours des années 20 par S. I. Bernštejn qui dirigeait le cabinet d'étude du langage littéraire à l'Institut d'histoire des arts. 
allemands : «O ritmomelodičeskih opytah prof. Sarana » (Les expériences rythmiques et mélodiques du professeur Saran). Toutefois Ejhenbaum, dans Melodika stiha (Mélodique du vers, 1922) et Tynjanov dans Problemy stihotvornogo jazyka (Problèmes du langage poétique, 1924) se gardèrent de reproduire à l'identique les idées de Sievers et de Saran. Ejhenbaum s'intéressait à l'unité de l'œuvre poétique, de ses intonations, de son sujet, impossible à saisir dans la reproduction orale, tandis que les philologues allemands avaient une approche linguistique, objectiviste de la poésie; Tynjanov, quant à lui, soulignait le caractère trop général de l'analyse phonétique des «philologues de l'audition » et croyait que la poésie ne pouvait être limitée aux questions orales (ainsi il prenait pour exemple le procédé des vers «manquants », marqués par des points de suspension, dans Eugène Oneguine, etc.) $)^{18}$.

- Enfin, du côté de la linguistique, et surtout de la stylistique, les formalistes furent marqués par l'école de Karl Fossler qui, pour une grande part, développa les idées de l'esthétique idéaliste de Benedetto Croce ${ }^{19}$. Les travaux de Fossler, dont une traduction russe parut dès 1910 dans la revue de philosophie de la culture Logos, développaient une idée humboldtienne (on peut dire néo-romantique) de la langue du point de vue de l'expression, envisagée comme un phénomène de vie nationale à une époque donnée, émanant d'un certain «milieu culturel ». Dans leurs recherches spécialisées en stylistique, les élèves de Fossler, au premier chef Leo Spitzer, développaient et concrétisaient cette idée d'étudier le style individuel d'un auteur comme s'il s'agissait d'une expression individuelle (littéraire) de la vie historique universelle (langagière) de telle ou telle nation, à telle ou telle période ${ }^{20}$. Cette conception était en gros familière à la philologie formaliste des années 1920, mais elle revêtit chez Vinogradov une forme très précise : il érigea en postulat l'idée qu'on ne pouvait étudier les particularités stylistiques de tel ou tel auteur qu'à la condition de les rapporter aux styles littéraires et, au-delà, aux réalités linguistiques de son époque.

Mihail Bahtin et son cercle (V. N. Vološinov, P. N. Medvedev) opposèrent systématiquement cette tradition allemande (subjectiviste), à laquelle ils souscrivaient en grande partie, à la tradition objectiviste française, attachée au nom de Ferdinand de Saussure ${ }^{21}$. Aujourd'hui on a une idée suffisamment claire des liens directs ou

18. Ju. N. Tynjanov, Literaturnyj fakt (Le fait littéraire), Moscou, Vysšaja škola, 1993, p. 3541.

19. Au sujet du lien entre Fossler et Croce, voir Iorgu Iordan, Romanskoe jazykoznanie (Lingvistica romanicaea), Moscou, Ed. du Progrès, 1971, p. 174-176.

20. M. P. Odesskij, «K voprosu o literaturovedčeskom metode L. Špitcera » (À propos de la méthode d'analyse littéraire de Leo Spitzer), in Teorija i praktika literaturovedčeskih i lingvističeskih issledovanij (Théorie et pratique des recherches en littérature et en liguistique), Moscou, 1988, p. 54-60.

21. Surtout dans le livre de V. N. Vološinov, Marksizm i filosofija jazyka (Le marxisme et la philosophie du langage, 1930) ; voir K. Brandist, « Političeskoe značenie bor’by s idejami Sossjura v rabotah školy Bahtina » (La signification politique de la lutte contre les idées de Saussure dans les travaux de l'école de Bahtin), Dialog, karnaval, hronotop, 2, 1995. 
indirects (y compris dans l'usage de termes tels que «phonème », etc.) entre les théories de Saussure et les idées de l'école linguistique de Moscou (Fortunatov et Poržezinskij), de Kazan (Kruševskij) et surtout de Saint-Pétersbourg (Baudoin de Courtenay) ${ }^{22}$. Toutefois, à la différence de l'approche psychologisante de Baudoin, la doctrine de Saussure se distinguait par son systémisme et sa quête de l'« antipsychologisme », selon l'expression de S. I. Bernštejn, qui fut l'un des premiers linguistes petrogradois à faire connaître les idées du savant genevois ${ }^{23}$. Rien d'étonnant si ce principe systémique du pionnier du structuralisme fut utilisé comme une arme par la philologie formaliste, qui visait la précision et l'objectivité dans l'étude de l'évolution littéraire (Vinogradov définissait même les travaux de Tynjanov comme une transposition des idées de Saussure dans le domaine des lettres). De ce point de vue, les formalistes eurent un allié de poids avec la naissance de la linguistique structurale ; or chez un Roman Jakobson, qui débutait alors ses recherches au sein du Cercle linguistique de Moscou, les deux sphères, linguistique et littéraire, n'étaient pas dissociables. Au sein de ce même cercle, A. I. Romm prépara au début des années 1920 une traduction du Cours de linguistique générale de Saussure, mais si Charles Bailly et Albert Sechehaye envisagèrent cette entreprise avec sympathie, ils finirent par refuser de la soutenir, sans doute pour ne pas contrarier les plans de S. I. Karcevskij 24 .

Outre les travaux des savants allemands déjà mentionnés, les formalistes furent aussi fortement marqués par le penseur danois Broder Christiansen (avec son principe de « qualité différentielle » comme écart esthétiquement signifiant par rapport à une norme universellement reconnue) et surtout par les idées d'Henri Bergson ${ }^{25}$ (dont la théorie antipositiviste, énergétique et téléologique de l'évolution aide à comprendre l'évolution de la littérature telle que la conçoit Šklovskij, et dont l'analyse du rire permet de comprendre la théorie de la parodie chez Tynjanov).

Cette interaction entre la philologie russe et européenne ne se déroulait pas dans un espace désincarné de pure filiation d'idées, mais dans un contexte politique tendu, voire dangereux, traversé de guerres et de révolutions qui, à partir de 1914, était dans l'ensemble peu propice au progrès de la coopération scientifique. Ces

22. A. A. Leont'ev, «Boduen i francuzskaja lingvistika» (Baudoin et la linguistique française), Izvestija AN SSSR. Serija literatury i jazyka, t. XXV, 4, 1966, p. 330-331.

23. «Iz istorii sovetskogo jazykoznanija. Rukopisnye materialy S. I. Bernštejna o F. de Sossjure » (Contribution à l'histoire de la linguistique soviétique. Matériaux inédits de S. I. Bernštejn sur F. de Saussure), publié par N. A. Sljusareva et V. G. Kuznecova, Izvestija AN SSSR. Serija literatury i jazyka, t. XXXV, 5, 1976, p. 445-448.

24. E. A. Toddes, M. O. Čudakova, «Pervyj russkij perevod "Kursa obščej lingvistiki” F. de Sossjura i dejatel'nost' Moskovskogo lingvističeskogo kružka » (La première traduction russe du Cours de linguistique générale de F. de Saussure et l'activité du Cercle linguistique de Moscou), in Fedorovskie čtenija 1978, Moscou, Nauka, 1981, p. 231-234. (Toujours au sein de ce cercle, A. K. Solov'eva, G. O. Vinokur, M. M. Kenigsberg et d'autres s'intéressèrent particulièrement à Saussure)

25. Pour plus de détails sur ce sujet, voir O. Hansen-Loeve, Russkij formalizm : Metodologičeskaja rekonstrukcija razvitija na osnove principa ostranenija (Le formalisme russe : une reconstruction méthodologique sur la base du principe de la défamiliarisation), Moscou, Jazyki russkoj kul'tury, 2001, p. 46-47 et 193-197. 
circonstances rendaient particulièrement important le rôle des «médiateurs »par lesquels passaient les liens directs entre les communautés scientifiques des pays européens - correspondances, voyages désormais plus rares, missions, contacts personnels, lettres de recommandation en vue de publications, etc. Dans la vieille génération c'étaient des philologues comme Fedor Andreevič Braun, doyen de la faculté d'histoire de la philologie à l'université de Saint-Pétersbourg au cours des années 1910, précepteur de Žirmunskij et d'Ejhenbaum, qui s'installa en Allemagne après 1920, où il devint avec Gorki coéditeur de la revue Beseda et professeur à l'université de Leipzig26 ; ou encore Lev Ščerba, étroitement lié à la linguistique française et, personnellement, à Antoine Meillet. Le linguiste russe Sergej Iosifovič Karcevskij (1884-1955) joua un rôle important en venant secourir les élèves directs de Saussure, Charles Bailly et Albert Sechehaye ${ }^{27}$. Pendant son séjour en Russie de 1917 à 1920 et jusqu'à sa nouvelle émigration en Suisse qui allait se prolonger jusqu'à sa mort, Karcevskij prit une part active à la Commission de dialectologie (il présenta notamment plusieurs communications aux « Entretiens sur la syntaxe » qu'elle avait organisés) et au Cercle linguistique de Moscou, la principale base du formalisme avec l'OPOJaZ de Petrograd ${ }^{28}$.

Parmi ces médiateurs, il convient de s'arrêter plus longuement sur Viktor Maksimovič Žirmunskij qui, au cours des années 1910, se passionnait de romantisme allemand et (avec Ejhenbaum) de philosophie esthétique ${ }^{29}$. Žirmunskij était particulièrement proche de la philologie allemande et les « corrections » qu'il apporta à la méthode formelle orthodoxe - la nécessité d'une analyse globale du style, la prise en compte du « sentiment de la vie » de chaque époque - s'inspiraient en droite ligne des idées de la Geistesgeschichte, et surtout d'Oskar Walzel. Žirmunskij fit traduire plusieurs œuvres de cet auteur en russe et, usant de son pouvoir de doyen de la faculté des lettres à l'Institut d'histoire des arts (GIII), il invita Walzel à Leningrad en automne 1928 ; il organisa d'autre part l'élection de Walzel, de Sievers et de Saran comme membres

26. A. I. Žerebin, « U istokov russkoj germanistiki (professor F.A. Braun) » (Aux sources de la germanistique russe: le professeur F. A. Braun), in Nemcy $v$ Rossii : russko-kul'turnye naučnye i kul'turnye svjazi, Saint-Pétersbourg, Dmitrij Bulanin, 2000, p. 14-21.

27. Voir l'article introductif d'Irène Fougeron à l'ouvrage $S$. I. Karcevskij, Iz lingvističeskogo nasledija (S. I. Karcevskij, Euvres linguistiques inédites), Moscou, Jazyki russkoj kul’tury, 2000.

28. R. O. Jakobson, « Moskovskij lingvističeskij kružok » (Le Cercle linguistique de Moscou), Ed. par M. I. Šapiro, Philologica, 3, 1996 ; G. S. Barankova, « K istorii Moskovskogo lingvističeskogo kružka : materialy iz Rukopisnogo otdela Instituta russkogo jazyka » (Contribution à l'histoire du Cercle linguistique de Moscou ; matériaux du Département de manuscrits de l'Institut de la langue russe), in Jazyk, kul'tura, gumanitarnoe znanie, Moscou, Naučnyj mir, 1999. Parmi les communications de Karcevskij à la Commission de dialectologie, on peut citer son exposé du 20 février 1918, d'esprit très « saussurien », sur le système du verbe russe, « Iz istorii sovetskogo jazykoznanija. Rukopisnye materialy S. I. Bernštejna o F. de Sossjure », op. cit., p. 441 .

29. E. G. Etkind, « Istorizm V. M. Žirmunskogo 1910-1920-e gg. » (L'historicisme de V. M. Žirmunskij, années 1910-1920), Izvestija AN SSSR. Serija literatury i jazyka, 1, 1952 (1992) ; G. M. Fridlender, «'Šestoe čuvstvo' (iz istorii literaturno-obščestvennyh nastroenij 1910-h gg. » (Le «sixième sens », contribution à l'histoire de l'atmosphère littéraire et politique des années 1910), Russkaja literatura, 2, 1992. 
honoraires de la faculté30. En 1924, il publia dans la nouvelle revue dirigée par Max Vasmer, Zeitschrift für slavische Philologie, un long article, dans lequel il faisait le bilan des travaux russes sur les questions de la forme en littérature ${ }^{31}$. L'année suivante, il se rendit en Allemagne ; parmi les fruits de ce séjour, il faut mentionner un article dans lequel il donnait un tableau des courants d'histoire littéraire en Allemagne ; cet article fut publié en 1927 dans un des numéros du recueil périodique Poetika. En outre, les éditions Academia publièrent en 1928, sous la direction de Žirmunskij, une anthologie intitulée Problema literaturnoj formy (Le problème de la forme littéraire), qui contenait des traductions d'auteurs allemands (Fossler, Walzel, Spitzer, Dibelius) et en 1929 la Soziologie der literarischen Geschmacksbildung de Levin Schücking, qui, à l'opposé des interprétations marxistes vulgaires de l'idéologie de classe de l'écrivain, étudiait les questions du mécénat et des goûts des lecteurs en littérature.

Cependant, au cours des années 20 , Žirmunskij, avec son engouement pour la science allemande bien établie et reconnue, passa de plus en plus aux yeux de l'aile orthodoxe du formalisme (à l'OPOJaZ) pour un épigone, un représentant de l'«éclectisme académique »32. L'attitude à l'égard de la philologie étrangère, notamment allemande, devint une pomme de discorde au sein du formalisme entre d'une part son trio de base (Šklovskij, Tynjanov, Ejhenbaum) et d'autre part son aile moscovite et son «école formelle philosophique » qui, après le départ de Jakobson pour la Tchécoslovaquie et la fin du Cercle linguistique de Moscou en 1924, se démarqua du futurisme et évolua de plus en plus vers l'esthétique néoclassique et une philologie traditionnelle orientée vers la philosophie, surtout sous l'influence de G. Špet à l'Académie des sciences de l'art (GANH). Au printemps 1927, Ejhenbaum prit connaissance des recueils qui venaient d'être publiés par les Moscovites et laissa dans son journal des preuves de son irritation :

« J'ai tâté aujourd'hui de la science moscovite. Moscou nous attaque : le recueil Hudožestvennaja forma (La forme dans l'art), le recueil Ars poetica. Mais comme tout cela est pitoyable, pauvre, prétentieux, suffisant ! Les références renvoient presque exclusivement à la science allemande, les théoriciens moscovites ne reconnaissent que Špet... et les Allemands. Ils n'ont pour nous que mépris, qu'ironie, comme si nous étions des enfants ! Quel toupet ! Pas une référence à Šklovskij, à Tynjanov, à moi. Une fois ou deux, on voit passer Žirmunskij, lui on peut, puisqu'il est 'doktor'. Alors qu'ils ont tout raflé chez nous, ces malotrus ! Alors qu'ils n'ont rien pour eux si ce n'est leur manière et une 'érudition' dont personne n'a besoin. »33

30. Gosudarstvennyj institut istorii iskusstv (L'Institut d'État de l'histoire des arts), Leningrad, 1927, p. 37

31. Zeitschrift für slavische Philologie, Bd. 1, H. 1-2, 1924, p. 117-152. Vasmer et Žirmunskij furent condisciples à l'université de Saint-Pétersbourg et collègues enseignants à celle de Saratov pendant la guerre civile. Il faut noter que Žirmunskij étudiait aussi la langue et le folklore des colons allemands en Ukraine.

32. K. Ju. Postoutenko, « Iz kommentariev k tekstam Tynjanova : 'akademičeskij eklektizm' » (Un commentaire aux textes de Tynjanov: l' «éclectisme académique»), in Sed'mye tynjanovskie čtenija. Materialy dlja obsuždenija (Septième rencontre Tynjanov. Matériaux pour une discussion), Riga - Moscou, 1995-1996.

33. Archives d'État de la Littérature et de l'Art (RGALI), f. 155, op. 1, d. 264 (Notes du 22 mars 1927). 
Sans doute Ejhenbaum était-il particulièrement irrité par l'article de R. O. Šor, « Formal'nyj metod na Zapade » (La méthode formelle en Occident) : il contenait des remarques sentencieuses à l'adresse des « erreurs très grossières » des formalistes russes, qui prétendaient avoir inventé une méthode scientifique, alors que, selon l'auteur, elle existait déjà depuis longtemps à l'étranger sous la forme d'une tradition scientifique structurée et éprouvée :

« Il n'y a guère de doute : dans la pratique de l'analyse formelle, en particulier celle de la composition, la science occidentale maîtrise parfaitement les méthodes et les procédés à la 'découverte' desquels prétendent les représentants de notre formalisme. [...] La tradition antique, avec laquelle les chercheurs occidentaux ont maintenu un lien parfaitement conscient, donne déjà de solides fondements pour systématiser les formes stylistiques. [...] Certainement, nos 'formalistes' devront aussi se rattacher à la même tradition, qui demeurait vivante dans la science et la critique littéraire russes au début du XIXe siècle. Il faudra surmonter la coupure qui avait été commencée par la critique philosophique et achevée par la critique publicistique au milieu du siècle dernier [...] comme le font les fondateurs de l'histoire de l'art - les symbolistes. »34

De façon générale, dans tout ce qui concernait leurs relations avec la science européenne, les formalistes eurent affaire, au début des années 1920, à deux sortes de reproches. On les accusait :

1) de vouloir devenir de «nobles étrangers », de considérer la culture russe comme si elle leur était étrangère, de la « défamiliariser », de vouloir la bousculer, en rompant avec la tradition de Belinskij, qui valorisait le lien organique entre l'art et la «vie » (voir, par exemple, les reproches d'Aleksandr Blok à Gumilev ou à l'Atelier des poètes (Ceh poetov), les critiques de type populiste et socialiste-révolutionnaire comme chez Ivanov-Razumnik et dans la Libre académie philosophique de Petrograd ou Vol'fila, ou encore chez G. P. Blok, de tendance conservatrice, dans sa correspondance avec B. A. Sadovskij);

2) de faire passer des évidences bien connues en Europe occidentale pour leurs dernières découvertes (outre Šor et le cercle des formalistes moscovites, on trouve ce genre d'accusations chez le critique bien connu A. Gornfel'd). Dans la revue Literaturnye zapiski parurent en été 1922 un billet satirique antiformaliste intitulé «Maksimalizm» (Maximalisme) et signé par le publiciste V. Ireckij, ainsi qu'une note de Gornfel'd, «Formalizm i ih protivniki » (Les formalistes et leurs adversaires) qui suscita aussitôt une violente protestation collective des formalistes, signée de Tynjanov, d'Ejhenbaum et de Tomaševskij ${ }^{35}$.

La vive réaction d'Ejhenbaum ainsi que des autres formalistes orthodoxes à ces références à la philologie occidentale était due en partie à son désir de défendre l'originalité de leurs propres découvertes scientifiques. Au début de l'année 1925, lorsqu'il commença à prendre ses distances avec le «formalisme d'école », ce qui

34. R. O. Šor, «Formal’nyj metod na Zapade » (La méthode formelle en Occident), Ars poetica, red. M. A. Petrovskij, Moscou, 1927, p. 136.

35. RGALI, f. 155, op. 1, d. 527,1.4-6. La réponse est restée inédite. 
allait le conduire plus tard à construire la théorie de la vie littéraire (literaturnyj byt), il relisait les travaux des philologues allemands et notait dans son journal : «Cela produit une impression étrange, comme si les Allemands avaient pris du retard sur nous. Ils en sont encore à s'embarrasser de vieux problèmes inutiles. » Et plus tard : «Comme tout est différent chez eux ! Le 'courant esthétique' (Walzel, Strich, Gundolf), c'est quelque chose que nous avons déjà dépassé. $»^{36}$ Dans une lettre à Šklovskij du début de l'année 1928, Tynjanov évoquait les travaux des formalistes en ces termes : « Nous nous sommes passés du Geist des Allemands et je crois que nous avons bien compris les choses. [...] Après nous, on ne pourra plus parler ni de mamaphore ${ }^{37}$, ni de Geist. $»^{38}$ Dans l'ensemble, selon nous, les formalistes avaient le droit de se considérer ainsi : la plupart des philologues allemands voyaient dans la littérature une incarnation de valeurs philosophiques et esthétiques qu'il fallait étudier sur le plan des techniques et des procédés littéraires, susceptibles d'être classés et catalogués pour peu qu'on s'appuyât sur la nomenclature antique traditionnelle des tropes et des figures rhétoriques. Le recours aux méthodes des historiens de l'art (Wölfflin et d'autres), la distinction, opérée à la suite de Simmel, entre le «vécu » et l'« expression », l'opposition entre l'esthétique et la forme des Temps Modernes, entre l'esthétique antique et la mesure, toutes ces novations de la philologie allemande de la fin du XIXe siècle ne changeaient pas le principe fondamental de l'analyse littéraire : la littérature ne devait pas être étudiée selon ses lois propres comme l'exigeaient les formalistes, mais demeurait l'expression de constantes qui lui étaient extérieures (le génie créateur et la volonté de l'auteur, la vie spirituelle de la nation, l'esprit du temps, etc.). Si on veut évaluer l'apport des formalistes russes, on peut dire qu'ils ont dans l'ensemble réussi à élaborer une approche plus historique et fonctionnelle, plus « contextualisante » de la littérature, que ne l'ont fait leurs collègues des autres pays (y compris la «nouvelle critique » anglo-américaine). Mais leur reconnaissance n'allait pas de soi, elle était tributaire des circonstances concrètes qui marquaient les études littéraires, la linguistique et la slavistique occidentales, et sur lesquelles nous allons nous pencher plus précisément à présent.

On peut résumer l'attitude des formalistes à l'égard de la philologie occidentale comme une indépendance croissante, accompagnée d'un certain désintérêt envers l'évolution des théories européennes dans le domaine littéraire ${ }^{39}$. Ils avaient pour cela de bonnes raisons, indépendamment même de leur originalité effective et de

36. B. Ejhenbaum, O literature, op. cit.,p. 511.

37. Jeu de mots parodiant les nomenclatures de la rhétorique.

38. Cité d'après Ju. L. Tynjanov, Poetika, istorija literatury, kino, op. cit., p. 536.

39. Mais ce serait une erreur de croire que les formalistes étaient indifférents aux réactions que leurs travaux suscitaient à l'étranger. Voici par exemple ce qu'écrivait Šklovskij à Tynjanov qui se trouvait à Berlin, le 5 décembre 1928, en discutant l'éventualité de reconstituer l'OPOJaZ : «Il faut prendre des contacts en Occident, s'assurer au moins de comptes rendus réguliers de nos articles. », «Iz perepiski Ju. Tynjanova i B. Ejhenbauma s V. Šklovskim (Extraits de la correspondance entre Ju. Tynjanov et B. Ejhenbaum avec V. Šklovskij), Voprosy literatury, 12, 1984, p. 194. 
leurs découvertes théoriques. L'étude de la littérature nationale est une pièce importante de la culture nationale, surtout depuis le début du XIXe siècle, à l'époque de la formation des États-nations européens, dont la légitimation passait entre autres par la construction de la tradition littéraire, de la culture des lettres, à la charnière desquelles on trouve la philologie et ses institutions ${ }^{40}$. Mais dans le cas présent, il s'agissait manifestement d'autre chose que de la conviction selon laquelle des chercheurs nationaux seraient seuls capables, en raison de leur « immersion » culturelle, participation et compétence, de comprendre correctement leur propres culture et littérature. Les philologues formalistes suivaient assez attentivement les travaux de leurs collègues à l'étranger (Šklovskij moins que les autres en raison de sa mauvaise connaissance des langues étrangères, de sorte que, pendant ses années d'émigration à Berlin en 1922-1923, il ne sortit pratiquement jamais des frontières du «Berlin russe $»^{41}$ ). En dehors de ce facteur, qui nous parait secondaire, la communication scientifique et culturelle s'effectuait en Europe dans des conditions radicalement nouvelles après la Première Guerre mondiale. Depuis la guerre des manifestes de l'automne 1914, puis de la participation de scientifiques des pays belligérants à des campagnes de propagande dirigées contre la culture des pays ennemis, la «république des lettres » européenne, avec ses pratiques habituelles de séjours et de stages dans les universités étrangères, laissa la place à un boycott de la science allemande et autrichienne de la part des organisations scientifiques internationales créées par les pays vainqueurs, y compris dans les sciences humaines ${ }^{42}$.

Dans ce contexte, le repliement sur soi de la science russe fut brutalement renforcé, à la charnière des années 20 et 30, par la lutte qui se déclencha contre les courants non marxistes dans le domaine des sciences humaines, en particulier contre les contacts avec l'émigration russe et les lecteurs occidentaux, contre la recherche de toute reconnaissance venant de l'étranger. Il faut noter particulièrement la discussion, par les élèves de Mihail Pokrovskij dans le cadre de la Société des historiens marxistes, des ouvrages de D. M. Petruševskij, Očerki iz ekonomičeskoj istorii Srednevekovoj Evropy (Essais d'histoire économique de l'Europe médiévale) et d'Evgenij Tarle, Evropa v epohu imperializma ( $L$ 'Europe à l'époque de l'impérialisme), et surtout ce qu'on a appelé l'«affaire Želebev », quand on accusa l'académicien S. A. Želebev d'avoir publié aux côtés de Mihail Rostovcev (académicien, émigré, cadet et opposant de principe au régime soviétique) dans le recueil Seminarium Kondakovium qui était édité à Prague ${ }^{43}$. Ce

40. Contribution à l'histoire des disciplines littéraires en France et Allemagne au XIX siècle, Michel Espagne et Michaël Werner, eds, Paris, Maison des sciences de l'homme, 1990.

41. Voir la lettre de V. B. Šklovskij à son oncle Dioneo (I. V. Šklovskij) écrite le 4 avril 1922 de Finlande, publiée par A. Ju. Galuškin, Tynjanovskij sbornik, Pjatye tynjanovskie čtenija (Recueil Tynjanov, Cinquième rencontre Tynjanov), Riga - Moscou, 1994, p. 285.

42. Cette question est examinée de façon approfondie dans B. Schroeder-Gudehus, Deutsche Wissenschaft und internationale Zusammenarbeit, 1914-1928, Genève, 1966, p. 113-133.

43. Voir Skifskij roman (Le roman scythe), red. G. M. Bongard-Levin, Moscou, Rosspen, 1997, p. 101-109. 
dernier épisode concernait directement les formalistes, car ils prenaient part, en même temps que Jakobson, à la revue Slawische Rundschau, également publiée à Prague. Jakobson dut s'assurer que la mission politique soviétique n'avait rien contre cette revue et aussi tranquilliser Šklovskij et Tynjanov au sujet de leur participation future ${ }^{44}$.

Cette conviction de leur propre originalité et d'une incompréhension potentielle de la part des représentants du savoir académique traditionnel, jointe à l'isolement croissant de la science soviétique, créa une distance avec la science occidentale, une sorte de «mur transparent » entre l'école formelle et les études littéraires européennes de cette époque, un mur qui était d'une autre espèce que l'enfermement naturel de toute tradition philologique nationale dans sa propre littérature ou culture. Très révélatrices à cet égard sont les pages du journal d'Ejhenbaum, dans lequel il décrit la visite du slaviste français André Mazon en URSS au début de l'année 1927 et les cérémonies «en trompe l'œil » que la direction de l'Institut d'étude comparée des littératures et des langues occidentales et orientales (ILJaZV - Institut sravnitel'noj istorii literatur i jazykov Zapada i Vostoka), N. S. Deržavin en tête, organisa en son honneur. Tout autre fut la soirée au cours de laquelle Ejhenbaum invita Mazon chez lui et qui devint une célébration informelle du dixième anniversaire de l'OPOJaZ. La communication qui y fut présentée par Tomaševskij fut publiée un an après par la Revue des Études slaves ${ }^{45}$ et devint le seul exposé «orthodoxe » et une sorte d'auto-interprétation de la doctrine formaliste en langue occidentale pendant tout le temps où cette école exista ${ }^{46}$. Un des fruits de la visite de Mazon fut sans doute également l'article de G. A. Gukovskij, en deux parties, sur Racine dans la Russie du XVIII siècle $^{47}$.

L'année 1930 fut celle de la fermeture de l'Institut d'histoire des arts, du « reniement » de Šklovskij, avec son article «Pamjatnik odnoj naučnoj ošibke » (Monument à une erreur scientifique ) dans Literaturnaja gazeta, et de l'interruption de fait des activités de l'école formelle. D'autre part, un de ses représentants les

44. Roman Jakobson : teksty, dokumenty, issledovanija (Roman Jakobson, textes, documents, études), Moscou, RGGU, 1999, p. 131 (Lettre de Jakobson à Šklovskij du 26 février 1929). Un certain nombre de scientifiques soviétiques collaboraient à la chronique bibliographique de cette revue : G. O. Vinokur, N. N. Durnovo, A. P. Skaftymov, etc. C'est là aussi que Tynjanov publia une critique élogieuse sur un recueil composé par T. I. Gric et M. I. Aronson, élèves d'Ejhenbaum, Kružki i salony (Cercles et salons), 1928.

45. B. Tomaševskij, « La nouvelle école d'histoire littéraire en Russie », Revue des Études slaves, VIII, 3-4, 1928, p. 226-240.

46. Nous ne comptons pas l'article déjà cité de Žirmunskij dans la Zeitschrift für slavische Philologie, car cet auteur nourrissait déjà alors une polémique active avec le formalisme « orthodoxe » de l'OPOJaZ. En 1927 on vit également paraître dans la revue de Vasmer cet article d'A. N. Voznesenskij : «Die Methodologie der russischen Literatursforschung in den Jahren 1910-1925 », ibid., 1927, Bd. 4, H. 1-2 et 1928, Bd. 5, H. 1-2. Voir également id., «Problems of method in the study of literature in Russia », Slavonic Review, 6, 1927.

47. G. Gukovskij, « Racine en Russie au XVIII siècle, I. La critique et les traducteurs », Revue des Études slaves, VII, 1-2, 1927 p. 75-93 ; «II. Les imitateurs », ibid., 3-4, p. 241-260. À noter que les premières publications de Gukovskij parurent en 1925 dans la revue de Vasmer et, probablement, sans le concours de Žirmunskij. G. Gukovskij, «Von Lomonosov bis Deržavin », Zeitschrift für slavische Philologie, Bd. 2, H. 2, 1925, p. 323-365. 
plus importants, Roman Jakobson, demeurait à l'étranger ; la même année, il publia un article à la suite du suicide de Majakovskij, «O pokolenii, rastrativšem svoih poetov » (Une génération qui a dilapidé ses poètes), qui lui ferma la route du retour et, pendant plus de deux décennies, lui interdit d'avoir des relations normales avec la science soviétique. Une étude du travail théorique et méthodologique auquel se livra Jakobson dans le prolongement de l'école formelle serait d'autant plus instructive qu'elle permettrait de considérer la reconnaissance scientifique internationale de cette école « à l'état pur », indépendamment de la présence du pouvoir soviétique et de son influence idéologique ou organisationnelle. L'attention principale devrait alors être portée sur la genèse de la méthode structuraliste, qui généralisait les principes des formalistes, au cours des années 20 et 30, dans le cadre du Cercle linguistique de Prague (CLP).

Jakobson vécut depuis 1920 en Tchécoslovaquie et, pendant les dix premières années, il maintint un lien étroit avec la représentation diplomatique soviétique qui put, grâce à lui, établir des relations avec différents milieux culturels ; elle s'appuya même sur lui pour une mission aussi délicate que l'invitation du président Masaryk en URSS par la voie scientifique et non pas diplomatique, à l'occasion du $200^{e}$ anniversaire de la création de l'Académie des sciences ${ }^{48}$. La commémoration de cette date à l'automne 1925 fut l'un des événements clés de la diplomatie culturelle soviétique naissante ${ }^{49}$ (sur l'exemple de Masaryk, on se proposa aussi d'inviter à ces festivités Paul Painlevé, mathématicien et académicien, et d'autre part premier ministre de France) $)^{50}$. Dans l'ensemble, depuis le début des années 20 , c'est avec l'Allemagne que la communauté académique soviétique eut les liens les plus étroits, car ces deux pays étaient tous deux parias du système diplomatique de Versailles et d'autant plus que, jusqu'à son adhésion à la Société des nations, l'Allemagne était pratiquement exclue des relations scientifiques internationales ${ }^{51}$. Après que l'Allemagne eut reçu en 1926 le droit de faire partie des organisations scientifiques internationales, réunies en 1919 en Conseil international de la recherche, ni elle, ni l'Union soviétique qui la soutenait ne profita de ce droit avant la fin de la Seconde Guerre mondiale, se limitant à des travaux conjoints dans différentes disciplines.

48. M. Ju. Sorokina, « 'Nenadežnyj, no absoljutno nezamenimyj' : 200-letnij jubilej Akademii Nauk i 'delo Masarika-Jakobsona' » («Peu fiable, mais absolument irremplaçable»: le $200^{\mathrm{e}}$ anniversaire de la création de l'Académie des sciences et « l'affaire Masaryk-Jakobson »), dans In Memoriam A. I. Dobkina (In memoriam d'A. I. Dobkin), Moscou, 2000.

49. Pour plus de détails, voir E. I. Kol’činskij, A. V. Kol’cov, «200-letnij jubilej RAN i politika » (Le 200e anniversaire de l'Académie des sciences de Russie et la politique), Naukovedenie, 1, 2000 .

50. Invitation qui ne put aboutir : la délégation française fut très peu nombreuse au regard de la délégation allemande, ce qui était dû en partie à un discours d'Antoine Millerand hostile à Ol'denburg à une séance de l'Académie des sciences morales et politiques et à un article inamical à l'égard de l'URSS dans le Journal des débats en juin 1925 : M. Ju. Sorokina, « Pridat'... impozantnyj harakter » (Prêter... un caractère imposant), Priroda, 12, 1999, p. 64-65.

51. Pour plus de détails, voir Sovetsko-germanskie naučnye svjazi perioda Vejmarskoj respubliki (Les relations scientifiques soviéto-allemandes pendant la République de Weimar), SaintPétersbourg, Nauka, 2001. 
La langue des publications demeurait un problème important. Le russe était la langue de la science soviétique, celle de l'émigration et d'une poignée (au regard du nombre de philologues et de spécialistes de sciences humaines en général) de slavistes occidentaux ${ }^{52}$. Par suite des mesures de boycott à l'encontre de l'Allemagne et de la perte de son rôle dominant dans bien des domaines scientifiques après la Première Guerre mondiale, l'allemand, langue traditionnelle des slavistes, cessa d'être la langue de communication scientifique qu'elle était devenue à la suite du latin au XVIII' et au XIXe siècle. Faut-il s'étonner dans ces conditions si la revue du Cercle linguistique de Prague prit le français pour langue de base, alors que la Slawische Rundschau, qui parut depuis 1929, était rédigée en allemand : au cours de cette période, le français supplantait efficacement l'allemand.

La Cercle linguistique de Prague commença à publier sa revue à l'occasion du Premier congrès mondial de linguistique qui se déroula à La Haye en 1928. De façon générale, le fonctionnement du Cercle qui faisait corps avec sa revue sur la base de thèses scientifiques militantes, cette forme de vie scientifique importée par Jakobson, se distinguait du style « individualiste » et tranquille qui régnait dans le monde universitaire en Tchécoslovaquie, plutôt périphérique au regard de l'Allemagne et de la France. Dans la correspondance de Jakobson et de Trubeckoj, l'offensive contre la science traditionnelle et l'affirmation des principes de la phonologie étaient planifiées comme un opération militaire. On trouve constamment des expressions comme «s'organiser », « mener une préparation » (provesti podgotovku), « les laisser s'exprimer, et ensuite leur coller une bonne dégelée » (vmazat' kak sleduet) dans ce document passionnant de la vie intellectuelle des années 20 et 30 ; elles visent, en règle générale, des personnes bien précises. Sans doute un des principaux adversaires du structuralisme nouveau-né était André Mazon, un adversaire du reste bien réel, rédacteur de la Revue des Études slaves et un des responsables de l'Institut d'études slaves à Paris. Mazon nourrissait des relations anciennes et solides avec des spécialistes de la littérature et des linguistes russes, il sut se gagner une réputation honorable par ses travaux sur Gončarov et Turgenev sur la base des collections parisiennes, il devint en 1928 membre-correspondant de l'Académie des sciences d'URSS et en 1935 membre de l'Institut de France. La visite de Mazon en Union soviétique, évoquée par Ejhenbaum dans son journal, se déroula dans des conditions plus officielles ${ }^{53}$ et bien plus favorables que celles de son voyage en 1918-1919. La première fois, alors qu'il rassemblait des publications actuelles pour la Bibliothèque nationale, il fut arrêté et passa plusieurs mois à la prison des Butyrki, mais il fut ensuite relâché et put emporter avec lui les

52. Voir la lettre extrêmement significative de N. S. Trubeckoj à P. N. Savickij du 8-10 décembre 1930 : O. A. Kaznina, « N. S. Trubeckoj i krizis evrazijstva » (N. S. Trubeckoj et la crise de l'eurasisme), Slavjanovedenie, 4, 1995, p. 93.

53. Au sujet des activités du Comité de coopération scientifique avec l'URSS, qui travaillait en étroite liaison avec le VOKS (Vsesojuznoe obščestvo kul'turnoj svjazi s zagranicej - Société pour les relations culturelles avec l'étranger) (et dont Mazon fut l'un des fondateurs et dirigeants), voir G. D. Kotova, «Iz istorii francuzsko-sovetskogo sotrudničestva (1925-1935 gg.) » (Une page de l'histoire de la coopération franco-soviétique, 1925-1935), Francuzskij ežegodnik 1975, Moscou, Nauka, 1977. 
collections rassemblées, grâce à l'aide de l'Académie des sciences et du commissariat du peuple aux Affaires étrangères ${ }^{54}$. L'un des résultats de cette visite fut son Lexique de la guerre et de la révolution en Russie (1920), que Jakobson accueillit par un compte rendu circonstancié et très critique dans la presse tchécoslovaque ${ }^{55}$. C'est sans doute de cette époque que date l'inimitié entre Jakobson et Mazon, qui ne put que se renforcer après le compte rendu plus que réservé que fit Mazon de l'ouvrage de Jakobson $O$ češskom stihe, preimuščestvenno $V$ sopostavlenii $s$ russkim (Le vers tchèque comparé principalement au vers russe) ${ }^{56}$. Mais cette polémique sortit au grand jour avec la publication des Travaux du CLP et lorsque commença l'audience internationale de la phonologie : il ne s'agissait plus ici d'une inimitié personnelle entre deux savants, mais d'un des épisodes centraux dans l'histoire de la réception du formalisme et du structuralisme en Europe. C'est pourquoi ce conflit mérite qu'on s'y arrête.

D'abord, dès ses débuts dans la carrière scientifique, Jakobson était porté aux généralisations, voire à un certain excès d'idées nouvelles et productives, commençant par établir des principes théoriques généraux, puis les appliquant à des matériaux concrets et très variés ; alors que Mazon, qui avait commencé comme historien de la littérature traditionnel et qui était ensuite passé à une étude de l'histoire des langues slaves anciennes, s'en tenait à des méthodes plus traditionnelles, positivistes et évolutionnistes modérées, fort éloignées d'un examen systémique des phénomènes linguistiques (y compris de la littérature), propre à Saussure et à ses héritiers. En particulier, lorsqu'il rendit compte des premiers Travaux du CLP dans la Revue des Études slaves, Mazon n'y trouva qu'un programme tonitruant, peu étayé par des matériaux concrets, alors que, selon lui, Antoine Meillet avait déjà proposé un développement semblable des idées de Saussure, mais exprimé dans une langue plus claire et plus simple ${ }^{57}$.

Ensuite, ce conflit était sous-tendu par une opposition entre des «partis » universitaires bien réels. Au sein de la communauté scientifique tchécoslovaque qui penchait justement vers l'influence française, par opposition à la forte et palpable influence germano-autrichienne traditionnelle, Jakobson s'orientait vers les concurrents parisiens de Mazon et des slavistes de l'Institut d'études slaves : l'historien Louis Eisenmann, rédacteur du Monde slave ${ }^{58}$ et Fuscien Dominois, qui

54. «Iz istorii sovetsko-francuzskih kul'turnyh svjazej » (Pages de l'histoire des relations culturelles soviéto-françaises), Istoričeskij arhiv, 3, 1961, p. 170-171.

55. Nove Atheneum, roc. II, sv. III, n $3,5,6$ et 7 .

56. Revue des Études slaves, III, 1-2, 1923, p. 144.

57. Revue des Études slaves, X, 1-2, 1930, p. 104 : compte-rendu d'une monographie de Jakobson, Remarques sur l'évolution phonologique du russe comparée à celle des autres langues slaves, publiée en 1929 au titre de second volume des Travaux du Cercle linguistique de Prague.

58. On trouve dans cette revue un article de Nina (Lazarevna) Gourfinkel, apparemment la sœur de M. L. Tronskaja (Gurfinkel`), élève très proche de Žirmunskij et traductrice de Walzel : «Les nouvelles méthodes d'histoire littéraire en Russie », Le Monde slave, 6, 1929, p. $234-263$ 
enseignait la langue et la littérature tchèques à l'École des langues orientales vivantes.

Enfin, ces divergences recouvraient des tensions culturelles et une distance entre la slavistique occidentale et son objet d'étude, problèmes auxquels Trubeckoj, le fondateur de la doctrine eurasienne, était particulièrement sensible. Très significatif à cet égard est cet extrait d'une lettre de Trubeckoj à Jakobson du 17 mars 1934, dans laquelle il décrit sa récente visite à Paris et son déjeuner en compagnie de Mazon et de ses collègues :

« Nous n'avons pas parlé de phonologie pour éviter de nous gâter l'appétit. En règle générale, la phonologie a bonne presse auprès des linguistes et mauvaise presse auprès des slavistes. Ils répètent toujours les mêmes sottises, s'indignent de la terminologie, etc. Il faut dire d'ailleurs qu'en dehors d'une antipathie personnelle à votre égard, il y a là également un rejet certain des formes de la culture eurasienne-danubienne dans lesquelles la phonologie moderne trouve son expression. On a beau dire, les slavistes français méprisent au fond de l'âme et prennent pour de la barbarie tout ce qui est slave, centre-européen et russe. Les savants slaves sont bons à réunir des matériaux, mais lorsqu'ils se prêtent à raisonner, ils découvrent leur manque de culture et leur âme slave ${ }^{59}$ : ce ne sont que fantaisies pures, esprit de secte, etc. C'est pourquoi un slaviste français n'admettrait jamais qu'un Russe ou un Slave lui enseigne quelque chose, du moins pas avant qu'il ne se soit francisé. C'est ainsi que j'explique pourquoi, précisément, les slavistes sont si opposés à la phonologie (en dehors des antipathies personnelles à votre égard, à cause de Dominois et d'Eisenmann), alors que les indo-germanistes et les autres linguistes n'ont rien contre elle. »60

Il serait trop simple d'expliquer ces jugements par le nationalisme de Trubeckoj ou par son ressentiment « anticolonial ». Justement, Jakobson et Trubeckoj avaient une attitude très positive à l'égard de la linguistique française, contrairement à leur jugement sur la linguistique allemande, qui était liée à Vossler et à la théorie des « milieux culturels $»^{61}$. En particulier, Trubeckoj admirait Meillet, dont il avait fait la connaissance à Sofia, et considérait que c'était un des meilleurs slavistes de l'époque. Il faut observer à ce propos que la théorie de Saussure, à laquelle se référaient les créateurs de la phonologie, était loin d'être au centre de l'intérêt et de l'attention de la France des années 20 et 30 (Jakobson et Greimas l'ont souligné après la Seconde Guerre mondiale) ${ }^{62}$. En 1929, Jakobson publia dans la Slawische Rundschau un article programmatique, dans lequel il se servait de la notion de « structuralisme » : « Les perspectives actuelles de la slavistique russe ». Il y opposait la tradition russe de l'analyse structurale et organique des objets des sciences humaines au positivisme platement historiciste occidental ; il ne s'agissait pas chez lui d'une quelconque supériorité originelle de la culture russe, mais d'une proxi-

59. En français dans le texte.

60. N.S. Trubetzkoy's letters and notes, La Haye - Paris, Mouton, 1975, p. 300-301.

61. Voir la lettre de Trubeckoj à Jakobson du 1er mai 1929, ibid., p. 131.

62. R. Jakobson, Izbrannye raboty (Travaux choisis), Moscou, Progress, 1985, p. 352. 
mité entre la tradition qu'il croyait voir en Russie et les nouvelles découvertes et recherches de la science mondiale ${ }^{63}$.

La polémique entre Jakobson et Mazon prit encore plus d'ampleur après que celui-ci eut publié, à la fin des années 30 , une série d'articles dans lesquels il exprimait des doutes sur la datation habituelle du Dit d'Igor et relevait la dépendance de ce texte à l'égard de la Zadonščina sur le plan de la langue et du style, alors que ce texte date, comme on le sait, de la fin du XIve siècle ${ }^{64}$. Plus tard, après la guerre, Jakobson et le byzantiniste belge Henri Grégoire publièrent aux États-Unis un ouvrage qui contenait une réfutation circonstanciée, écrite dans un style très agressif, des thèses de Mazon ${ }^{65}$. Boris Ungebaun, élève de Mazon, et qui partageait l'avis de Jakobson sur le fond de l'affaire, écrivait à ce dernier dans une de ses lettres :

«Quant aux observations qu'il fait sur le ton de votre recherche, il s'agit d'un malentendu qui tient à des traditions différentes dans la critique littéraire. Le ton de votre polémique, parfaitement habituel dans les républiques d'Europe centrale, est en effet assez rare dans la pratique française, et c'est pourquoi il suscite la surprise du lecteur français. Or j'ai déjà eu l'occasion d'observer des réactions similaires: des personnes qui sont choquées par le ton que vous employez, commencent à éprouver insensiblement une certaine sympathie envers Mazon, même lorsqu'elles ne partagent pas ses vues. ${ }^{66}$

Certainement, dans la longue et complexe histoire de la reconnaissance du formalisme ou du structuralisme pragois par la science européenne, il ne saurait être question d'expliquer ce qui précède par l'arrogance de Jakobson, ni par le caractère hautain de Mazon, ni par de quelconques traits de caractère qui, de toute façon, ne pouvaient qu'aggraver une situation objectivement tendue et contradictoire. Les principes de la nouvelle phonologie furent d'abord formulés dans le contexte d'une tradition scientifique périphérique, dans une période où le statut des sciences, y compris des sciences humaines, était en pleine évolution, aussi bien que leur image

63. Roman Jakobson : teksty, dokumenty, issledovanija, op. cit., p. 31-33.

64. André Mazon, Le Slovo d'Igor, Paris, 1940. Voir également « Perepiska S. I. Karcevskogo i R. O. Jakobsona » (Correspondance entre S. I. Karcevskij et R. O. Jakobson), publ. par H. Baran et E. V. Dušečkina, dans Roman Jakobson : teksty, dokumenty, issledovanija, op. cit., p. 175-191. Pavel Miljukov s'insurgea également en 1937 contre 1'hérésie de Mazon dans la presse émigrée.

65. La geste du prince Igor, épopée russe du XII siècle, New York, 1948. Cette édition fut préparée à l'Institut de philologie et d'histoire orientale et slave, à l'École libre des hautes études, fondée par les gouvernements français et belge en exil. C'est dans les murs de cette école que Jakobson, qui y enseigna de 1942 à 1946 la linguistique générale et la philologie slave, fit la connaissance de Claude Lévi-Strauss.

66. H. Baran et E. V. Dušečkina, « Vokrug 'Slova o polku Igorove' : iz perepiski R. O. Jakobsona i A. V. Solov'eva » (Autour du Dit d'Igor : une correspondance entre R. O. Jakobson et A. V. Solov'ev), Slavjanovedenie, 4, 2000, p. 72, n. 3 (Lettre d'Unbegaun à Jakobson du 5 juillet 1948). 
sociale et culturelle ${ }^{67}$. Jakobson et son école furent reconnus après la guerre aux États-Unis, grâce à ses percées en linguistique ${ }^{68}$ et c'est seulement pourquoi ses découvertes en poétique scientifique gagnèrent également la notoriété. Le formalisme dut attendre les années 60 pour gagner après coup, en premier lieu grâce aux efforts de Jakobson, une image franchement positive, à mesure que s'affirmait le structuralisme, dont il apparaissait comme un prédécesseur semi-légendaire, et que la linguistique et les méthodes exactes en sciences humaines, qui sortaient des cadres traditionnels des études littéraires, faisaient l'objet d'un intérêt général. C'est alors seulement que le formalisme cessa de n'être qu'une école de critique littéraire à moitié oubliée et circonscrite à un des pays slaves, pour devenir un courant extrêmement important des sciences humaines du Xxe siècle. L'analyse concrète de cette nouvelle évolution (le rôle et la chronologie des différentes anthologies formalistes qui parurent dans différentes langues européennes ${ }^{69}$, l'importance de l'école sémiotique de Moscou - Tartu qui devenait déjà connue à ce moment-là, la réaction des pionniers du formalisme qui étaient encore en vie (Šklovskij, Jakobson), autant de questions qui mériteraient des recherches spécialisées, mais qui sortent du cadre de cette étude.

(traduit du russe par Wladimir Berelowitch)

Institut d'histoire des sciences et des techniques,

Section de Saint-Pétersbourg, Académie des sciences

aldmitr@eu.spb.ru

67. V. V. Ivanov, « Iz istorii rannih etapov stanovlenija strukturnogo metoda v gumanitarnyh naukah slavjanskih stran » (Les débuts de la méthode structurale dans les sciences humaines des pays slaves), in Moskovsko-tartuskaja semiotičeskaja škola. Istorija, vospominanija, razmyšlenija (L'école sémiotique de Moscou - Tartu. Histoire, mémoires, réflexions), Moscou, 1998. Au sujet des conditions spécifiques de cette lutte pour la reconnaissance de la théorie structuraliste en linguistique et en poétique dans une région périphérique de la slavistique, voir également A. Žolkovskij, in Roman Jakobson : teksty, dokumenty, issledovanija, op. cit., p. 277.

68. Voir R. Jakobson, K. Pomorska, Besedy, op. cit., p. 32-38.

69. La première et la principale de ces anthologies fut publiée en français par Tzvetan Todorov et Roman Jakobson, Théorie de la littérature. Textes des formalistes russes, Paris, Le Seuil, 1966 